\title{
The adaptation of colorectal cancer cells when forming metastases in the liver: expression of associated genes and pathways in a mouse model
}

Derya Bocuk', Alexander Wolff', Petra Krause ${ }^{1}$, Gabriela Salinas³, Annalen Bleckmannn ${ }^{2,4}$, Christina Hackl ${ }^{5}$, Tim Beissbarth ${ }^{2}$ and Sarah Koenig ${ }^{1,6^{*}}$

\begin{abstract}
Background: Colorectal cancer (CRC) is the second leading cause of cancer-related death in men and women. Systemic disease with metastatic spread to distant sites such as the liver reduces the survival rate considerably. The aim of this study was to investigate the changes in gene expression that occur on invasion and expansion of CRC cells when forming metastases in the liver.

Methods: The livers of syngeneic C57BL/6NCrl mice were inoculated with 1 million CRC cells (CMT-93) via the portal vein, leading to the stable formation of metastases within 4 weeks. RNA sequencing performed on the Illumina platform was employed to evaluate the expression profiles of more than 14,000 genes, utilizing the RNA of the cell line cells and liver metastases as well as from corresponding tumour-free liver.

Results: A total of 3329 differentially expressed genes (DEGs) were identified when cultured CMT-93 cells propagated as metastases in the liver. Hierarchical clustering on heat maps demonstrated the clear changes in gene expression of CMT-93 cells on propagation in the liver. Gene ontology analysis determined inflammation, angiogenesis, and signal transduction as the top three relevant biological processes involved. Using a selection list, matrix metallopeptidases 2, 7, and 9, wnt inhibitory factor, and chemokine receptor 4 were the top five significantly dysregulated genes.

Conclusion: Bioinformatics assists in elucidating the factors and processes involved in CRC liver metastasis. Our results support the notion of an invasion-metastasis cascade involving CRC cells forming metastases on successful invasion and expansion within the liver. Furthermore, we identified a gene expression signature correlating strongly with invasiveness and migration. Our findings may guide future research on novel therapeutic targets in the treatment of CRC liver metastasis.
\end{abstract}

Keywords: Colorectal cancer (CRC), RNA-sequencing, Gene expression, Liver metastasis

\footnotetext{
* Correspondence: koenig_sarah@ukw.de

'Department of General, Visceral and Paediatric Surgery, University Medical

Centre, Georg - August - University Goettingen, Göttingen, Germany

${ }^{6}$ Medical Teaching and Medical Education Research, University Hospital

Wuerzburg, Julius-Maximilians-University Wuerzburg, Josef-Schneider-Str. 2/

D6, 97080 Wuerzburg, Germany

Full list of author information is available at the end of the article
}

(c) The Author(s). 2017 Open Access This article is distributed under the terms of the Creative Commons Attribution 4.0 International License (http://creativecommons.org/licenses/by/4.0/), which permits unrestricted use, distribution, and reproduction in any medium, provided you give appropriate credit to the original author(s) and the source, provide a link to the Creative Commons license, and indicate if changes were made. The Creative Commons Public Domain Dedication waiver (http://creativecommons.org/publicdomain/zero/1.0/) applies to the data made available in this article, unless otherwise stated. 


\section{Background}

Colorectal cancer (CRC) is the third most common type of cancer in the Western world and the second most common cause of cancer-related death in both genders. The overall relative 5-year survival of CRC patients is approximately $50 \%$ [1]. Almost half of all patients suffering from CRC are confronted with liver metastasis either at the time of diagnosis (15 to $20 \%$ ), or later during the course of the disease (25\%) [2]. Given the rather poor 5-year survival rate of patients who develop liver metastasis (approx. 30\%), it is vital that we develop and evaluate new therapeutic strategies. In particular, the knowledge of molecular changes to CRC cells that end up in the liver may enable us to search for new target options far more selectively.

Metastasis is frequently a final and fatal step in the progression of solid malignancies. The nature and time of onset of the changes that provide tumour cells with metastatic functions are still largely unknown. Furthermore, there has been an ongoing debate to this end for more than a 100 years. In 1889, Stephen Paget noticed that the pattern of metastases produced by different neoplasms was not random. In his 'seed and soil' hypothesis, Paget claimed that certain tumour cells ('seeds') have an affinity for the microenvironment of specific organs ('soil'), and only when the 'seed' and the 'soil' are compatible can metastasis occur [3].

With respect to the "seed", it is widely accepted these days that cancer is attributed to the accumulation of genetic alterations in cells. Thus, to understand the molecular mechanisms of cancer metastasis, it is indispensable to identify not only the genes whose alterations accumulate during cancer progression but also those genes whose expression is responsible for the acquisition of metastatic potential in cancer cells [4]. Indeed, comparative analyses of the gene expression profiles of metastatic and non-metastatic cells have revealed that various genes are differentially expressed in association with the metastatic potential of cancer cells [4]. Conversely, the existence of genes expressed by rare cellular variants that specifically mediate metastasis has been disputed [5]. Transcriptomic profiling of primary human carcinomas has identified gene expression patterns that, when present in the primary tumour, predict a poor prognosis for patients $[6,7]$. The existence of such signatures can be interpreted in the sense that genetic lesions acquired early on in tumorigenesis may prove sufficient for the metastatic process, and that consequently no metastasisspecific genes exist.

There is growing evidence that the development of or progression to metastases is also dependent on the "soil". Tumour cell circulation, extravasation into a distant organ, angiogenesis, and uninhibited growth also provide essential hints as to the metastatic process [8].
The molecular requirements for some of the steps involved may be highly tissue specific. For example, the proclivity that tumours have for specific organs, such as breast carcinomas for bone and lung, was noted more than a century ago [9]. Moreover, the potential of tumour cells to metastasize depends on their interaction with homeostatic factors in the target organ that promote tumour-cell growth: survival, angiogenesis, invasion, and progression. It seems that the intrinsic cellular heterogeneity within tumour populations evolves through an extrinsic selection process, which is based on more or less infrequent cellular variants with augmented metastatic abilities and which finally mediates the outgrowth in distant sites [9]. Of note, the mechanism that enables the liver microenvironment to influence the behaviour of CRC cells is still only poorly understood.

The most common site for CRC metastasis is the liver [10]. Many patients still suffer from recurrence of the primary and/or distant metastasis, even after undergoing liver resection combined with adjuvant approaches such as chemo- and radiotherapy. Nonetheless, only a minority of patients actually survive for years [11]. Therefore, the a priori or early inhibition of metastasis could prove to be a key step towards the curative treatment of patients. We have to assume that each organ places different demands on circulating cancer cells for the homing and subsequent outgrowth of metastases.

To clarify this issue, we established a novel syngeneic and orthotopic mouse model of CRC liver metastasis. This model comprises the injection of cells from a known CRC cell line to mimic the spread of the primary tumour and thus to investigate the invasion and expansion of CRC cells in the liver on the gene expression level. The goal here was to identify genes that contribute to this process of adapting to the new "soil" and thereby the metastatic progression of the disease. The fundamental aim of the study was to identify new candidate markers or molecular mechanisms in the diagnosis of liver metastasis resulting from CRC, as well as therapeutic targets effectively inhibiting CRC metastasis in the liver.

\section{Methods}

\section{Reagents and antibodies}

Unless specified otherwise, all chemicals and reagents were supplied by Life Technologies (Darmstadt, Germany). Foetal Bovine Serum Superior (FBS) was purchased from Biochrom (Berlin, Germany) and trypsin 10-fold was supplied by PAA (Pasching, Austria). Antibodies for immunolabelling purposes were purchased and used as illustrated in Table 1.

\section{Cell lines and culture}

The cell line CMT-93 (isolated from a mouse colorectal adenocarcinoma) was kindly donated by Christina Hackl 
Table 1 Antibodies used in immunolabelling analysis

\begin{tabular}{lllll}
\hline Antigen & Species & Dilution & Catalogue & Manufacturer \\
\hline B-catenin & Rabbit & $1: 50$ & $14-6765$ & eBioscience, Frankfurt a.M., Germany \\
CD44 & Rat & $1: 1000$ & 550,538 & BD Pharmingen, Heidelberg, Germany \\
Ki-67 & Rabbit & $1: 200$ & $275 R-14$ & Cell Marque, California, United States \\
E-cadherin & Rabbit & $1: 50$ & sc-7870 & Santa Cruz Biotechnology, Heidelberg, Germany \\
Vimentin & Rabbit & $1: 1000$ & ab92547 & Abcam, Cambridge, UK \\
Anti-rat biotinylated & Donkey & $1: 200$ & RPN1004 & GE Healthcare, Freiburg, Germany \\
Avidin HRP & & $1: 400$ & 18-4100-94 & eBioscience, Frankfurt a.M., Germany \\
HRP Labelled anti-rabbit & Goat & Ready to use & K4002 & Dako, Hamburg, Germany \\
\hline
\end{tabular}

and her workgroup in Regensburg, Germany. On testing, the cells were found to be negative for mycoplasma by RT-PCR.

CRC cells were expanded and stored in frozen aliquots $\left(-70{ }^{\circ} \mathrm{C}\right)$. After thawing, the cells were routinely cultured in $75 \mathrm{~cm}^{2}$ culture flasks in DMEM high glucose, supplemented with $10 \%$ FBS, $1 \%$ L-glutamine, $1 \%$ sodium pyruvate and $1 \%$ penicillin/streptomycin at $37{ }^{\circ} \mathrm{C}$ and $5 \% \mathrm{CO}_{2}$ in a humidified incubator. Tumour cells were passaged once (following 3 days in culture), cultured for a further 4 days, and then trypsinized for subsequent implantation studies. Tumour cells from the same passage were used for all the implantation experiments. Additionally, aliquots of the cell line were snap frozen and processed for transcriptome sequencing analysis (RNA-seq).

\section{Animals and procedures}

Ten-week-old female C57BL/6NCrl mice (mass 18-22 g) were purchased from Charles River (Sulzfeld, Germany). Animals were kept on a 12-h day/night rhythm and fed with a phytoestrogen-reduced mouse diet (ssniff, Soest, Germany).

Prior to (surgical intervention) surgery, animals received a subcutaneous application of carprofen (Rimadyl ${ }^{\circ}$, Pfizer, Berlin, Germany) (5 mg/kg body mass). Animals were anaesthetized under constant sevoflurane inhalation (Sevorane ${ }^{\oplus}$, Abbott, Wiesbaden, Germany). After median laparotomy, the hilum of the liver was exposed to access the portal vein. One million tumour cells in a volume of $100 \mu \mathrm{l}$ PBS buffer were injected slowly into the portal vein using a $30 \mathrm{G}$ needle.

In the study group, seven animals were implanted with tumour cells. The control group encompassed five animals which underwent the same procedures (sham-OP), but were only injected with buffer solution. All animals were sacrificed after 4 weeks. Explanted livers were sliced for macroscopic assessment, photographic documentation of the section planes, and further processing. Tissue samples from the tumour core of the liver metastases derived from CMT-93 as well as matched unharmed liver tissue (macroscopically tumour-free liver) were excised, snap frozen for whole transcriptome sequencing analysis (RNA-seq), or frozen in 2methylbutane at $-70{ }^{\circ} \mathrm{C}$ for immunolabelling.

\section{Immunolabelling}

Cryostat sections $(5 \mu \mathrm{m})$ were fixed in ice-cold acetone for $10 \mathrm{~min}$ and were stored at $-80{ }^{\circ} \mathrm{C}$. After rehydration in Tris/ $\mathrm{HCl}$ buffer $(\mathrm{pH}$ 7.6), sections were incubated with the primary antibodies (see Table 1) overnight at $4{ }^{\circ} \mathrm{C}$. Endogenous peroxidase was inactivated by incubation with $0.3 \% \mathrm{H}_{2} \mathrm{O}_{2}$ in $70 \%$ methanol and $30 \%$ Tris/ $\mathrm{HCl}$ buffer for $20 \mathrm{~min}$ at RT. The HRP-labelled goat anti-rabbit IgG secondary antibody (DakoCytomation K4002, Carpinteria, USA, ready-to-use reagent) was used to identify $\beta$-catenin, Ki-67, E-cadherin, and vimentin. To immunolabel CD44, sections were exposed to an avidin/ biotin blocking step (Life Technologies, Darmstadt, Germany) followed by incubation with the primary antibody (overnight at $4{ }^{\circ} \mathrm{C}$ ). This antigen was identified by the secondary antibodies donkey anti-rat biotinylated (1:200, $1 \mathrm{~h}$ at RT) and avidin-horseradish peroxidase (HRP) (1:400, $1 \mathrm{~h}$ at RT). 3-amino-9-ethyl-carbazole (AEC) solution (BD Pharmingen, Heidelberg, Germany) and haematoxylin counterstaining were used for visualization by light microscopy. Negative controls were carried out for each antibody by omitting the primary antibody from the protocol. Samples were covered with $50 \mu \mathrm{l}$ of the aqueous mounting agent Aquatex (Merck, Darmstadt, Germany) and evaluated under a light microscope (LEICA DM IRE2, Bensheim, Germany).

\section{RNA isolation}

For RNA sequencing purposes (RNA-seq), three aliquots of the cell line and specimens (tumour core and liver) from seven animals were collected. The RNA purification system PeqGold TriFast (Peqlab, Erlangen, Germany) was used to isolate RNA from metastatic liver tissue. Briefly, specimens were defrosted in peqGold TriFast (1 ml/100 $\mathrm{mg}$ tissue) and then homogenized using TissueLyser LT (Qiagen, Hilden, Germany) at 
$50 \mathrm{~Hz}$. Total RNA was isolated according to the manufacturer's instructions and stored at $-80{ }^{\circ} \mathrm{C}$. In addition, the High Pure RNA Isolation Kit (Roche, GrenzachWyhlen, Germany) was used to isolate RNA from CMT93 cells according to the manufacturer's recommendations. The quantity and integrity of the isolated RNA was assessed in a NanoDrop ND - 1000 spectrophotometer, version 3.5.2 (Peqlab, Erlangen, Germany), using the $260 \mathrm{~nm} / 280 \mathrm{~nm}$ absorbance ratio and was further analysed with an Agilent 2100 BioAnalyzer (Agilent Technologies, Santa Clara, California, USA) as a quality check. RNA-seq was performed at the Transcriptome and Genome Analysis Laboratory in Goettingen, Germany, using an Illumina HiSeq2000 sequencer (Illumina, Inc., San Diego, California, USA).

\section{Deep sequencing analysis}

As starting material for the library preparation, $0.5 \mu \mathrm{g}$ of total RNA was used. The libraries were generated according to the TruSeq mRNA Sample Preparation Kits v2 Kit from Illumina (Cat. $\left.N^{\circ} R S-122-2002\right)$. The fluorometric based QuantiFluor ${ }^{\mathrm{Tu}}$ dsDNA System from Promega (Mannheim, Germany) was used for accurate quantitation of cDNA libraries. The size of final cDNA libraries was determined by using the Fragment Analyzer from Advanced Bioanalytical. cDNA libraries were amplified and sequenced by using the cBot and HiSeq2000 from Illumina (SR; $1 \times 50 \mathrm{bp}$; ca. 30 Mio reads per sample). Sequence images were transformed to bcl files using Illumina software BaseCaller, which were demultiplexed to fastq files with CASAVA v1.8.2 and quality checks were done via fastqc.

\section{Statistics}

\section{Preparation of data/statistical model}

An in-house RNA-seq analysis pipeline employing the STAR-aligner (version 2.4.0 h) [12] for the mapping and counting of reads with the expectation-maximization algorithm implemented in the software package RSEM (version 1.2.19) [13] was used for counting reads. Ensembl Mus musculus GRCh38 Version 78 was considered as the reference for mapping and further annotations.

Following RNA-seq, all seven tumour probes derived from CMT-93 underwent quality control measures. Employing the corresponding RNA-seq data, they were checked for the expression of CK 20 as surrogate parameter for colorectal tissue or liver-specific gene expression to identify liver-specific genes, such as phosphoenolpyruvatecarboxykinase 1 (PCK1), cytochrome p450 (CYP), and carbamoyl phosphate synthase1 (CPS1). Three tumour probes (D213K, D214K, D215K) representing false biopsies were excluded from further analysis owing to a strong infiltration of liver (approx. 4 to 20 times the elevated expression levels of liver enzymes) and low content of colorectal tissue.

Principal component analysis (PCA) was performed in $\mathrm{R}$, the programming language and environment (version 3.2 ), to visualize the underlying structure of the dataset by calculating the eigenvectors and plotting those two components with the highest variance in the data.

Focussing on the comparison between the cell line and metastases, we filtered out differential genes specific to liver tissue, which we considered as 'liver tissue effect'. Thus, differentially expressed genes (DEGs) were identified as either up- or down-regulated when comparing the CMT-93 cell line with the unharmed liver tissue. Subsequently, these differences relating to the normal liver background were excluded from the gene expression results between the cell line and liver metastases. This filtering step was done in order to identify genes representing differences in cell line versus tumour, instead of general differences in cell lines versus normal liver.

\section{Significant differential gene analysis}

Basing on the read counts attained from RSEM, the $R$ package EdgeR [14] was used to calculate the mean intensities as well as the $p$-value and the log fold change (logFC) for each DEG, comparing the CMT-93 cells with the liver metastases formed. Thus, gene differences between the two groups were identified by fitting a negative binomial generalized linear model implemented in EdgeR. Expression results were reported as mean transcripts per million (TPM) values for each group.

A list was created comprising 119 genes associated with metastasis, based on the genes described in the Tumor Metastasis RT2 Profiler PCR Array by Qiagen Hilden, Germany (Additional file 1). This list was applied as a filter following completion of the analysis of the DEGs to profile the expression of these genes in our dataset.

\section{Gene ontology (GO) analysis}

A gene set was defined, comprising all the DEGs identified in the comparison of CMT-93 cells and the liver metastases that formed, corrected for the liver background and with a false discovery rate of less than $5 \%($ FDR < 0.05). This gene set was employed in the gene ontology and pathway analysis. This method, implemented in the $\mathrm{R}$ package topGO [15], allows us to identify GO terms that are over-represented (or under-represented) using the annotations for that gene set taken from the Gene Ontology Database (http:// www.geneontology.org/). The significant level of GO terms for the DEGs was analysed with the weighted Fisher's exact test in the package. We computed $p$-values for all the 
DEGs in the GO category "biological processes"; the threshold of significance was defined as $p$-value $<0.05$.

\section{Results}

Syngeneic mouse model of CRC metastasis in the liver Following the injection of CMT-93 cells via the portal vein and their subsequent expansion, $70-80 \%$ of animals developed liver metastases in any number of liver lobules during the course of the study, as seen on macroscopic assessment (Fig. 1a). Within the life span of the animals, CMT-93 cells had colonized about $30-50 \%$ of the mouse liver with the tumour spots increasing to approximately 5 to $10 \mathrm{~mm}$ in diameter. However, the spread of the tumour burden resulting from CMT-93 proved to be inhomogeneous when comparing the right and left liver lobules of injected mice. On the microscopic level, immunohistochemical staining 4 weeks after tumour cell injection into the portal vein was used to assess phenotypic expression of colorectal carcinoma markers. Liver metastases displayed features of a moderately differentiated colorectal adenocarcinoma revealing complex glandular structures (40-75\% gland formation) in a desmoplastic stroma (Fig. 1 b, c). An important feature of invasion is the presence of this desmoplasia or desmoplastic reaction, a type of fibrous proliferation surrounding tumour cells and secondary to the invasive tumour growth. The glandular structures expressed epithelial markers such as membrane-bound $\beta$-catenin (Fig. 1d) and E-cadherin (Fig. 1e). The latter was expressed mostly in the cytoplasm, which indicates that this wnt marker was inactive. The hyaluronic acid receptor CD44 (a putative marker of 'stemness' in CRC) was also present and staining was detected in nearly all of the tumour cells we investigated (Fig. 1f). The unsystematic arrangements of gland formations also expressed the mesenchymal marker vimentin (Fig. 1g). The proliferation marker Ki-67 was expressed abundantly in more than $75 \%$ of all liver tumours in a random pattern (Fig. 1h).

\section{Adaptation of CMT-93 cells when forming metastases in the liver}

To assess the factors involved in the formation of liver metastases, we performed RNA-seq analysis on both the liver metastases as well as unaffected liver tissue. Figure 2 summarizes the structure of the gene expression data. The first principal component $(\mathrm{PC})$ is plotted on the $\mathrm{x}$ axis and captures $74 \%$ of the variance. The second $\mathrm{PC}$ is plotted on the $y$-axis and captures $19 \%$ of the variance. The PCA plot clearly portrays the separation of the CMT-93 cell line samples from those of the corresponding macroscopically tumour-free liver, as well as from the derived liver metastases. As assumed, the cluster associated with CMT-93 was found to be located in close proximity to the cluster relating to the derived

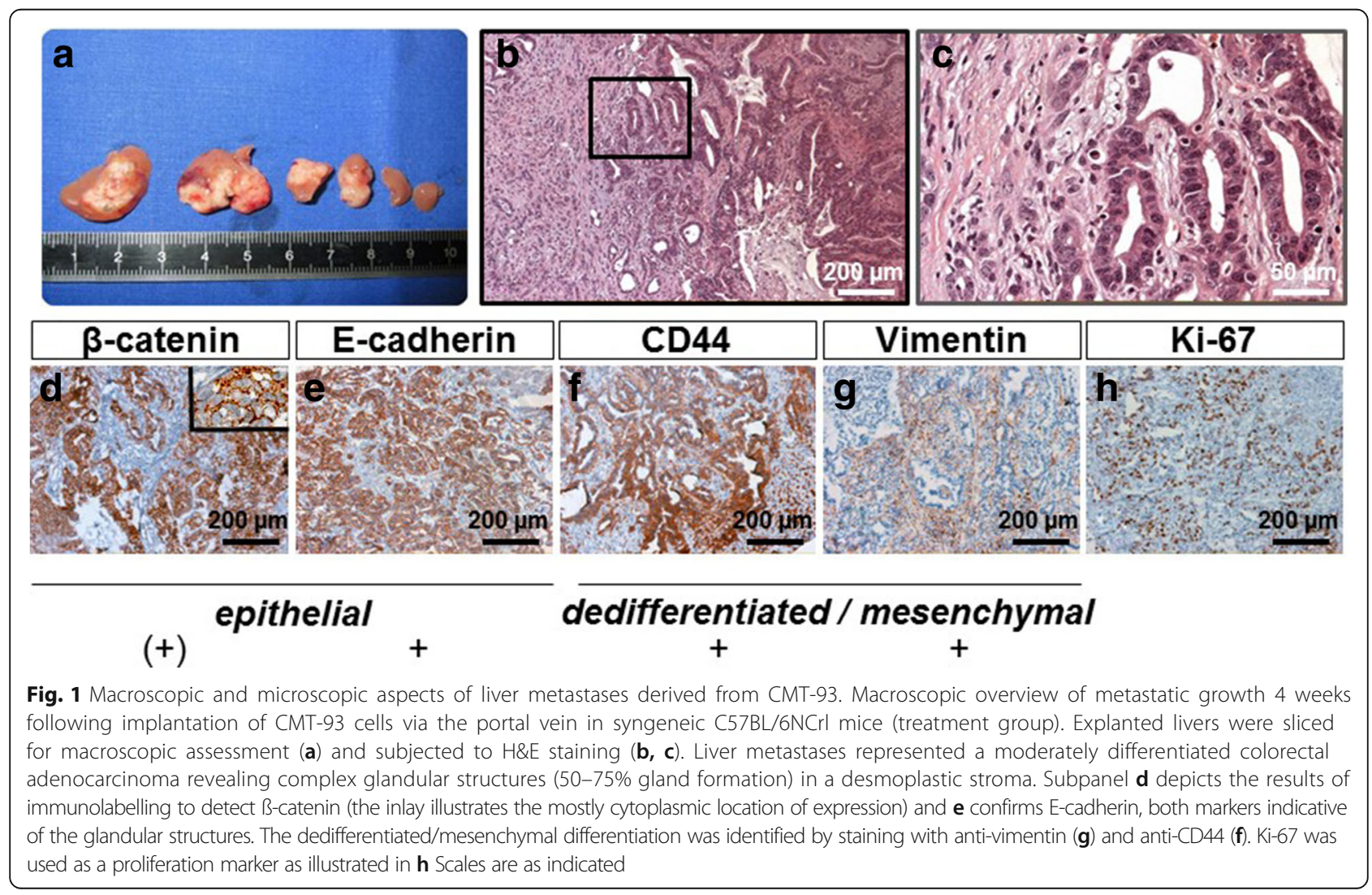




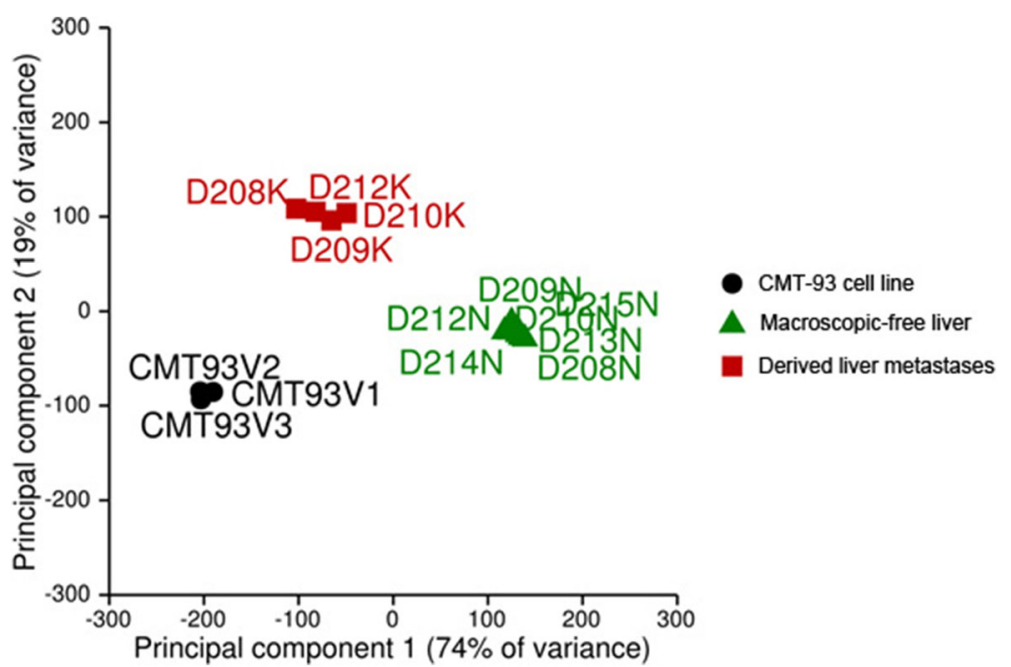

Fig. 2 PCA was used to visualize the underlying structure of the dataset. CMT-93 cells as well as tumour probes (tumour liver tissue) formed clusters clearly separate from the corresponding macroscopically tumour-free liver. Each group was tightly clustered

metastases. We also plotted liver specimens originating from sham-operated animals (injection of buffer alone). When overlaid on the PCA plot in Fig. 2, these samples lay in exactly the same position as the cluster of the macroscopically tumour-free liver samples (data not illustrated).

Table 2 lists the DEGs during the propagation of CMT-93 cells in the liver. A total of 5297 genes were down-regulated and 6597 were up-regulated when CMT-93 cells propagated in the liver. The elimination of DEGs relating to the liver background (see materials and methods) reduced the total number of DEGs to 1174 down-regulated genes (35\%) and 2155 up-regulated genes (65\%).

The results of hierarchical cluster analysis to assess the relatedness of the 120 DEGs displaying the greatest differences in expression are presented in Fig. 3. The heat map reveals systematic and fairly clearly distinguished variations in the expression of genes between the original CMT-93 cells, the derived liver metastases, and tumour-free liver. Of note, the changes in expression of the CMT-93 cells outgrowing as liver metastases is clearly apparent. However, the hierarchical clusters relating to the metastases and the tumour-free liver are somewhat closer to each other owing to the fact that the implanted CMT-93 cells forming metastases infiltrate the liver tissue. The gene expression profile is therefore bound to reflect the colonization of the hepatic tissue.

We then applied a filter set of 119 selected genes associated with metastasis to our RNA-seq data set following the elimination of DEGs determining the liver background. Thus, 32 relevant genes were identified with a threshold of FDR $<5 \%$, of which 23 were up-regulated and 9 down-regulated. Additional file 2 contains the count data of the samples. Figure 4 illustrates the heat map of the filtered DEGs within the CMT-93 cell line and the liver metastasis samples. Hierarchical clustering confirmed the clear changes between DEGs of the CMT-93 cells and the derived metastases. Table 3 presents an overview of these genes which were also ranked by $p$-value. The top five dysregulated genes were found to be matrix metallopeptidase (MMP) 7, keratin 20 as an epithelial marker of colorectal carcinoma, wnt inhibitory factor 1, MMP 9, and chemokine receptor 4 .

With respect to functional gene groups, the 23 upregulated genes were attributed to extracellular matrix proteins ( 6 genes), cell growth and proliferation (5 genes), cell adhesion (4 genes), wnt signalling (3 genes), transcription factors/regulators and epithelial-mesenchymal transition (EMT) (2 genes each), and CRC-related genes (1 gene). The 9 down-regulated genes were assigned to cell growth and proliferation, wnt signalling and

Table 2 Differences in gene expression among the sample groups (DEGs)

\begin{tabular}{lllll}
\hline & CMT-93 vs. liver metastases & $\begin{array}{l}\text { Macroscopic-free liver vs. } \\
\text { liver metastases }\end{array}$ & CMT-93 vs. macroscopic-free liver & $\begin{array}{l}\text { CMT-93 vs. liver metastases } \\
\text { corrected for the liver background }\end{array}$ \\
\hline$-1=$ down-regulated & 5297 & 5163 & 5764 & 1174 \\
$0=$ unregulated & 3667 & 4157 & 2684 & 0 \\
$1=$ up-regulated & 6597 & 5749 & 6187 & 2155 \\
\hline
\end{tabular}




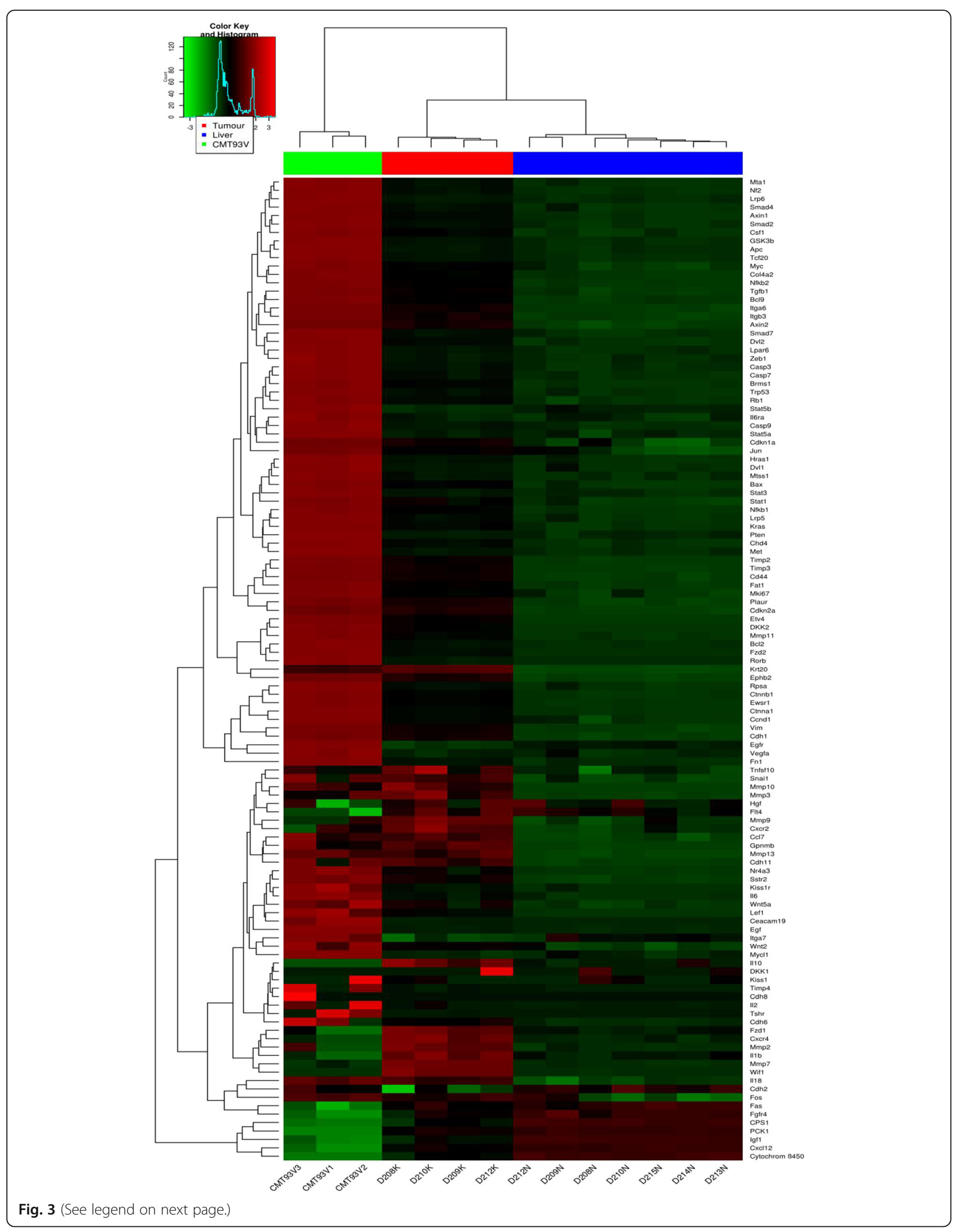


(See figure on previous page.)

Fig. 3 Top 120 DEGs between CMT-93 cells, liver metastases derived from CMT-93 and macroscopically tumour-free liver. Expression data are depicted as a data matrix in which each row represents a gene and each column represents a sample. The colour coding bar above the heat map marks the samples from the CMT-93 cell line (samples CMT93V1-3) as green, those from the liver metastases derived from CMT-93 (samples D208-210 K, D212K) in red and those of macroscopically tumour-free liver (samples D208-210 N, D212-215 N) in blue. Expression levels are depicted according to the colour scale presented in the top left corner. Red indicates expression levels above and green below the median, respectively. The magnitude of deviation from the median is represented by the colour saturation. The hierarchical clustering is visualized by the dendrogram at the top, which illustrates the degree of relatedness in gene expression

transcription factors/regulator (2 genes each), as well as apoptosis genes, extracellular matrix proteins, and EMT (1 gene each).

\section{Biological processes involved}

To address the pathways and processes involved, significant DEGs with FDR $<5 \%$ were selected and tested against the background set of all genes with GO annotation (Fig. 5). The most relevant GO terms for biological processes enriched were "inflammatory response", "angiogenesis", "signal transduction", "positive regulation of transcription from RNA polymerase II promoter", "transmembrane receptor protein tyrosine kinase signalling pathway", and "positive regulation of ERK1 and ERK2 cascade".

\section{Discussion}

CRC is a common disease whose considerable metastatic potential highlights the urgency and necessity to develop novel therapeutic approaches to prevent or treat tumour progression and metastasis. In this study, we set out to analyse the changes in gene expression and pathways that play a role in the colonisation of mouse liver by the cell line CMT-93, mimicking the processes that lead to the ultimate formation of liver metastases secondary to CRC. To this end, we first had to establish the in vivo metastatic mouse model. CMT-93 cells demonstrate a strikingly efficient tumorigenic capacity following their implantation via the portal vein, the common route CRC cells take when colonising the liver. Furthermore, the CMT-93 cell line originated from the mouse strain we employ, C57BL/6NCrl; the two are thus syngeneic and this enables us to circumvent rejection responses as complications. Moreover, the mice are immunocompetent, which allows us to investigate the normal inflammatory response to tumour growth. Of note, the outgrowing metastases in this model are reproducible and display a number of prototypic features (structure and markers) common to human CRC liver metastases. The model itself acts as a paradigmatic proof of principle for genetic alteration when forming liver metastases. A xenograft model involving human CRC cell lines would not have been able to fulfil our criterion of immunocompetence to mimic the seed and soil theory in humans. While searching for suitable models we tried three combinations in total, based on literature research and commercial availabilities. Although CT-26 (ATCC CRL-2639 $9^{\text {ma }}$ ) and the mouse strain Balb/c resulted in tumour formation in the liver, these were found to be mesenchymally de-differentiated in nature. The cell line APC1638-NT (kindly donated by Prof. R. Smits, Rotterdam, Netherlands) with mouse strains C57BL/6 $\mathrm{N}$ or C57BL/6 J resulted in no detectable tumour growth whatsoever (data not shown). The CMT-93/C57BL/ $6 \mathrm{NCrl}$ model proved to be the only one demonstrating reproducible liver metastatic growth with the histological features of colorectal cancer.

Disseminating tumour cells need to adapt to surrounding tissues in a continuous fashion [16]. For example, CRC cells of the primary tumour have to avoid succumbing to any immune response, detach from the primary, migrate into the portal system, arrive in the liver, traverse the endothelial barrier of the portal vessels (extravasation), overcome hypoxia on integration into the liver parenchyma, adapt to the new environment, initiate angiogenesis, and finally expand as metastases (metastatic colonisation) [17-19]. Taken together, these numerous influences during the process of metastatic spread into clinically detectable macroscopic disease lead to marked changes in gene expression. This new gene signature is the result of a multi-step process in which carcinoma cells progress along the "invasion-metastasis cascade" [18]. The results of our study visibly support the notion that CRC cells certainly undergo a number of clear changes in the liver environment.

It goes without saying that every single finding from the dataset following RNA-seq analysis cannot be commented on in this highly specific context during propagation in the liver. However, there are some definite hints as to which genes and pathways have to be addressed when aiming to treat CRC liver metastasis.

Our RNA-seq expression analysis and subsequent filtering with the selection list of the most-relevant metastasis-related genes reveals that the liver environment stimulates CMT-93 cells into expressing a number of genes enhancing metastasis. These genes are associated with functions such as tissue remodelling, cell proliferation, adhesion, wnt activity, transcription/ regulation, and inhibition of apoptosis, which all contribute to metastatic activity and tumour cell invasion. 


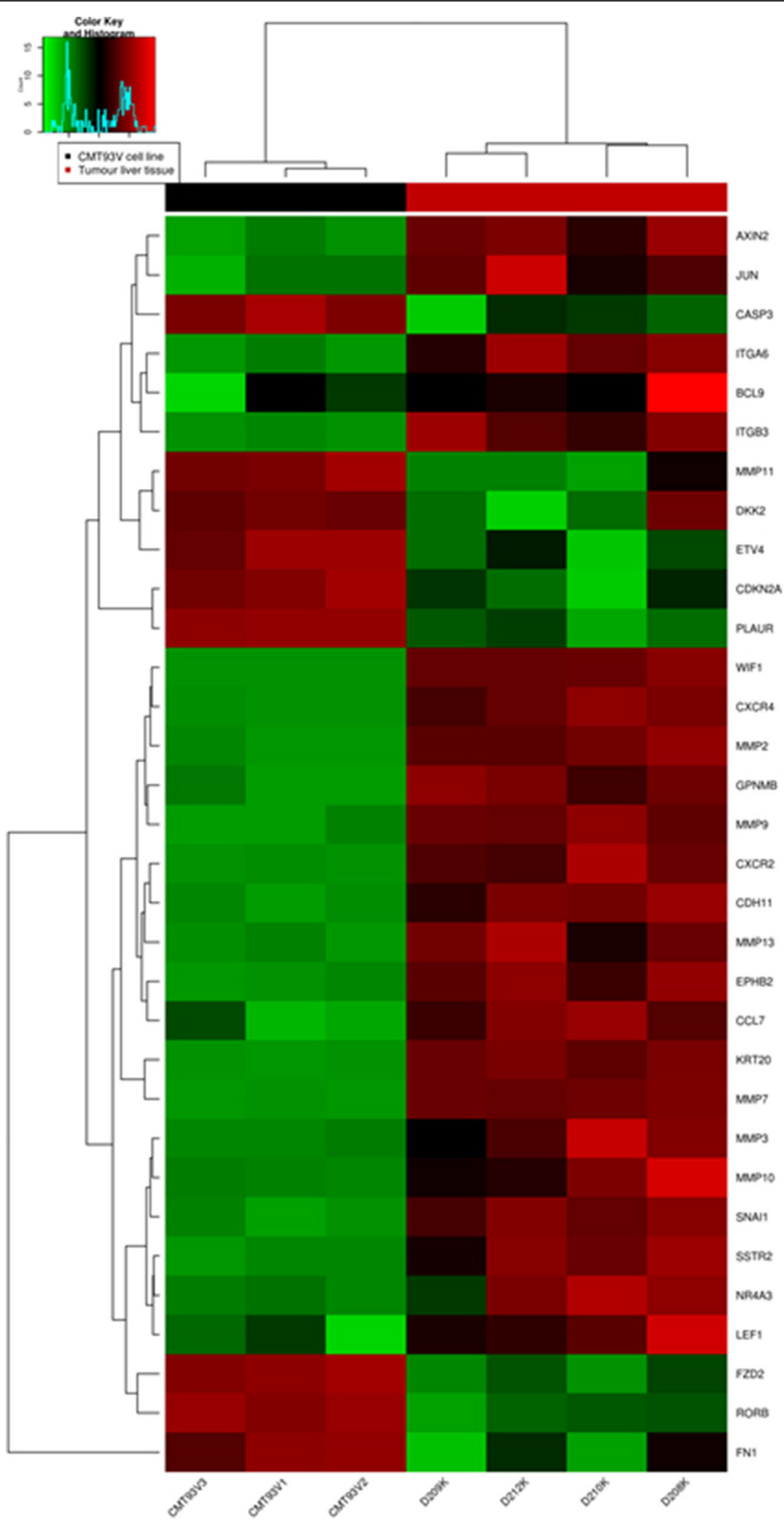

Fig. 4 Heat map illustrating the expression signature of 32 genes related to liver metastasis development. Unsupervised analysis was performed on the data set using our filtered gene list (119 genes associated with metastasis). Depicted are 32 representative genes which were expressed differentially during the propagation of CMT-93 cells in the liver 


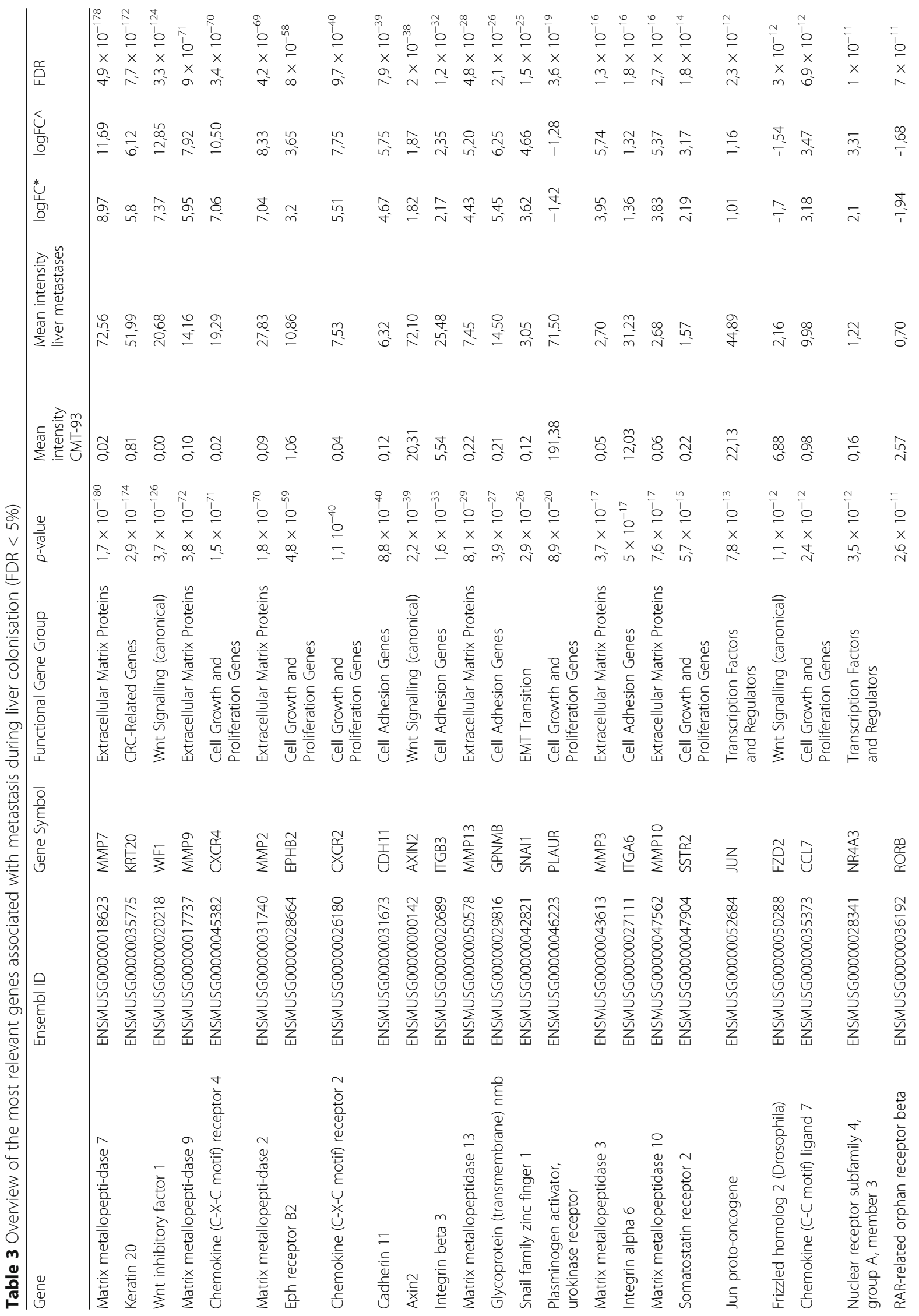




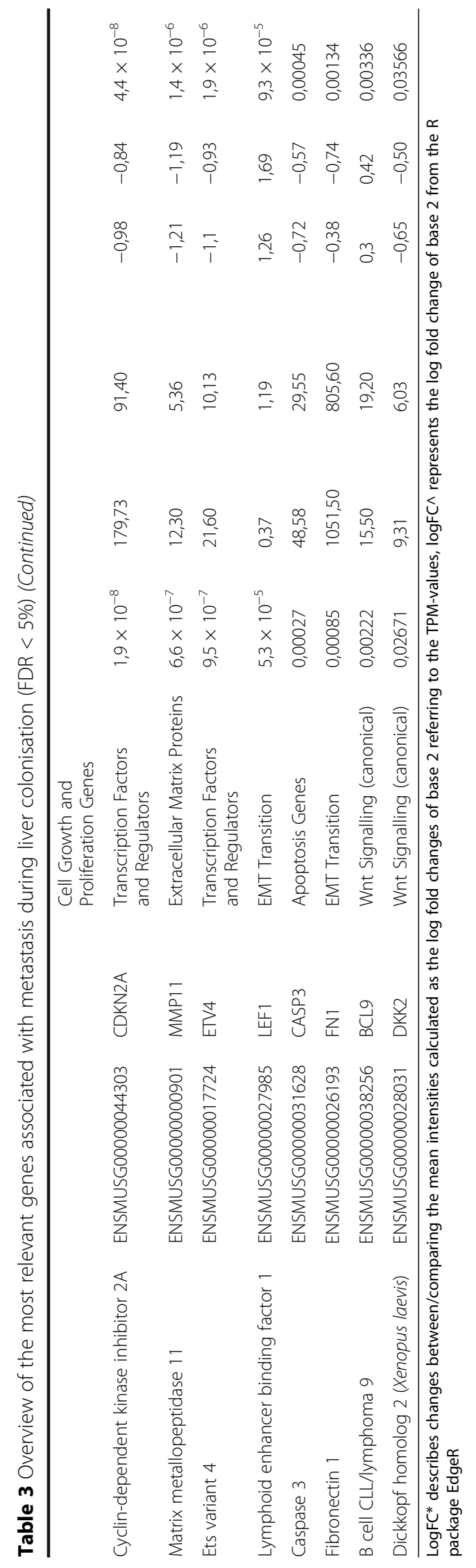




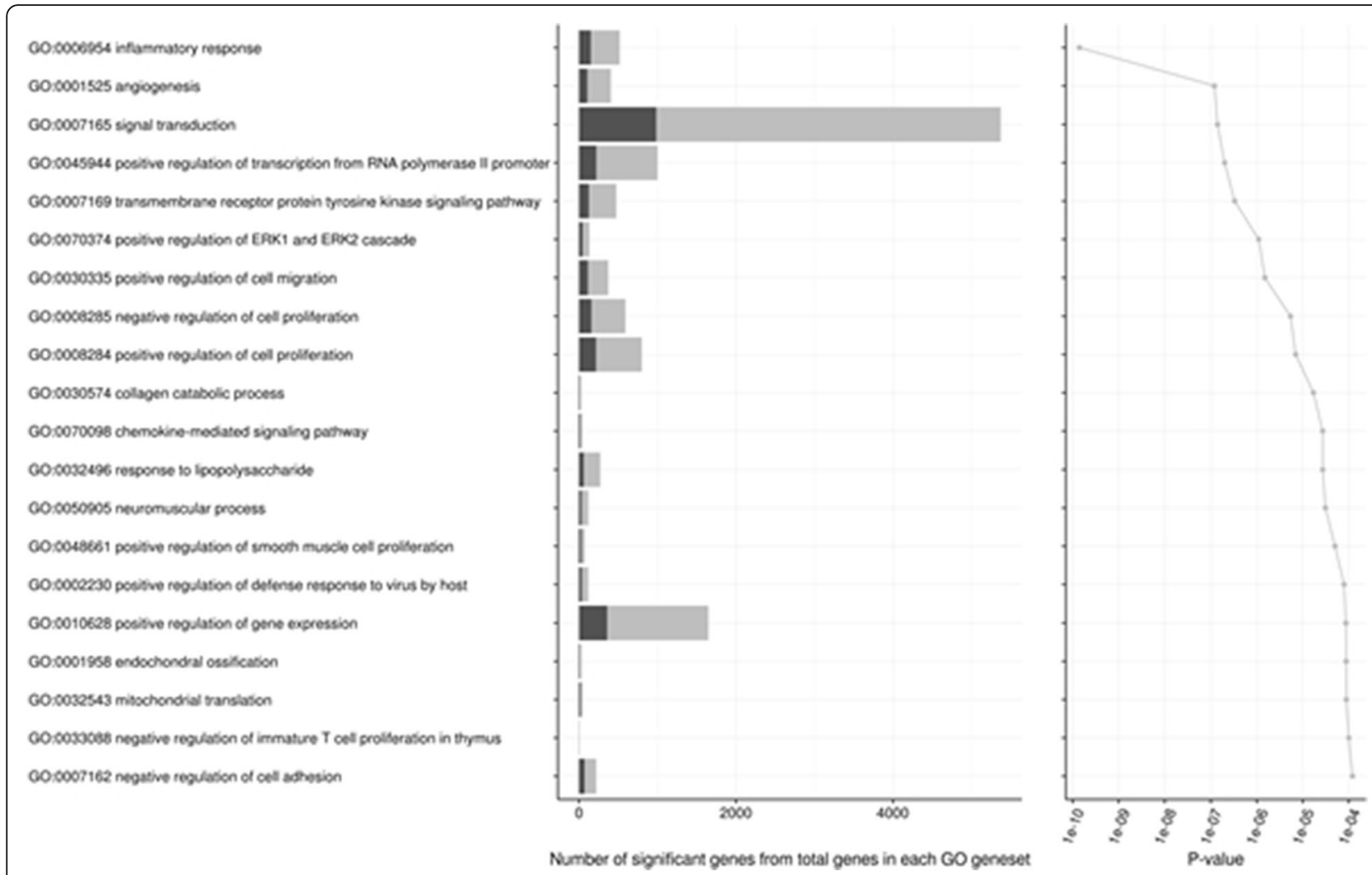

Fig. 5 Bar plot of GO pathways. Top 20 Gene Ontology categories from biological processes comparing the CMT-93 cell line with the liver metastases derived thereof. GO categories are depicted and sorted by $p$-value. The dark grey bar indicates the number of significant genes from all genes in that GO category (dark and light grey). Together with the light grey region of the bar, the plot summarises the total number of genes on the pathway

Specifically, MMPs, chemokine receptors, and integrins are predominantly up-regulated in liver metastases derived from CMT-93.

Interactions between carcinoma cells and stromal cells play a vital role during the invasion of the new anatomic metastatic site. Tumour cells must first traverse the basement membrane and then create space for further expansion. Components of the extracellular matrix (ECM) contain a repository of growth factor molecules that can be liberated by proteases secreted by carcinoma tissue. Moreover, the basement membrane also plays crucial roles in signal transduction events within carcinoma cells via pathways initiated by integrin-mediated cell-matrix adhesions, leading to alterations in cell polarity, proliferation, invasiveness, and survival [20]. Additionally, the entry of CRC cells into the hepatic microvasculature can also initiate the pro-inflammatory cascade that results in Kupffer cells being triggered to secrete chemokines [21]. Those are known to up- and also down-regulate various vascular adhesion receptors, thereby enabling adhesion of CRC cells in the microvasculature of the fibroblasts and myofibroblasts, endothelial cells, adipocytes, and various bonemarrow-derived cells - including macrophages and other immune cells [22].
GO analysis is widely recognized as the premier tool in the organization and functional annotation of molecular aspects of cellular systems [23]. We determined the significant GO categories based on a threshold of significance of $p<0.05$. Our results reveal that the GO terms inflammatory response, angiogenesis, and signal transduction were the most relevant biological processes involved in the propagation of CRC in the liver. Most recently, Becht et al. reported that CRC molecular subgroups and micro-environmental signatures were highly correlated [24]. More precisely, he stated that the mesenchymal subtype of CRC was characterized by a high density of fibroblasts that most likely produces the chemokines and cytokines which favour tumour-associated inflammation and support angiogenesis, resulting in a poor prognosis. Looking into features of assessable inflammatory state in patients, Hamilton et al. were able to link elevated serum levels of $\mathrm{C}$ - reactive protein (CRP) with increased circulating pro-inflammatory cytokines. Those patients with colorectal liver metastases were attributed with shorter disease-free and overall survival following surgical resection [25]. Most recently, the inflammatory milieu of CRC liver metastases was used to investigate a new treatment option based on TIE2- 
expressing monocytes/macrophages (TEMs), a myeloid cell subset. Adopting the concept of gene transfer, TEMs located in peritumoral sites and exerted an anti-tumour effect through the release of interferonalpha (IFN $\alpha$ ) [26]. Utilizing this strategy in mouse models of CRC liver metastasis, TEMs accumulate in the proximity of hepatic metastatic areas and the TEM-mediated delivery of IFN $\alpha$ inhibits tumour growth. In our study, we could not detect IFN $\alpha$ as being deregulated to a significant level in the liver metastases. However, there were a number of DEGs associated with interferon (e.g. interferon-activated gene 205 (IFI205), interferon-induced transmembrane protein 1 (IFITM1) and interferon gamma inducible protein 30 (IFI30)), which were not members of the top 100 list (data not shown).

Different angiogenic factors have been related to metastasis formation because they promote primary tumour growth and increase the likelihood that tumour cells come into contact with blood and thus disseminate [27]. In particular, the liver is known to be a permissive soil with respect to angiogenesis. The liver parenchyma adjacent to the synchronous liver metastases provides an angiogenically favourable environment for metastatic tumour growth [28]. On the individual level, there was significant correlation between primary CRCs and matched liver metastases with respect to vascular endothelial growth factor (VEGF) mRNA expression. VEGF mRNA levels in patients with two or more liver metastatic tumours were significantly higher than those in patients with only solitary liver metastases [29]. To date, oxaliplatin- and irinotecan-based chemotherapy regimens combined with monoclonal antibody treatment in the form of bevacizumab (anti-VEGF) have proved to be efficient as first-line therapy of metastatic colorectal cancer $[30,31]$.

Invasion processes are crucial to the formation of liver metastases in CRC and regularly involve a variety of MMPs leading to the degradation and remodelling of the extracellular matrix (ECM) $[18,27]$. CRC liver metastases express MMP7 more intensely than normal liver [32]. Our results support the notion that MMP7 is one of the significant players enhancing invasiveness in CRC [33-35]. It is worth noting here that targeted therapy in this matter is difficult. There is evidence in some preclinical models that MMP inhibitors (MMPIs) are effective at multiple stages of CRC tumour progression, inhibiting both establishment and growth of primary CRC tumours, as well as reducing metastasis in the lungs and liver [36]. However, clinical trials with MMPIs have been largely unsuccessful as therapeutic agents in CRC so far. A recent study in pre-clinical mouse models of metastatic CRC suggests that ulinastatin (an intrinsic trypsin inhibitor) and natural polyphenol curcumin are capable of inhibiting CRC liver metastases via modulation of MMP9 and E-cadherin expression [37].

The model developed certainly proved to be suitable, as the invasion and expansion of CMT-93 following their injection via the portal vein leading to liver metastasis was reproducible. Our results clearly support the notion of an invasion-metastasis cascade with notable changes to the expression profile of CMT-93 cells on entering and expanding in the liver. Although we were perhaps able to shed some light on the bigger picture, the relative importance of distinct events, interactions, and the molecular drive that all serve to facilitate organspecific colonisation will require further investigation.

\section{Conclusions}

Our work demonstrates that the gene expression in tumour cells is clearly altered during and following the process of metastasis. Here, bioinformatics greatly assists in the analysis of large amounts of data derived from RNA-seq. Through rigorous experimental planning and sophisticated statistical analysis, we are a step closer to elucidating the factors and processes involved during the liver metastasis of CRC. One or more of these dysregulated genes may prove to be a worthy target and enable us effectively to switch off a CRC cell's capacity to act as seed in the formation of metastases. Such a development, at best during the early stages of disease progression, for example prior to the outgrowth of tumour cells within the target soil, could well have a markedly positive effect on the prognosis as well as overall survival of CRC patients.

\section{Additional files}

Additional file 1: Supplement 1. List of 119 genes associated with metastasis. The dataset was filtered using this list to identify DEGs between the CMT-93 cell line and liver metastases derived from CMT-93. Gene names and Ensembl IDs are shown in Additional file 1: Supplement 1. (XLSX $11 \mathrm{~kb}$ )

Additional file 2: Supplement 2. Count data of relevant genes for the propagation of CMT-93 cells in the liver. Thirty-two DEGs were identified with a threshold of FDR $<5 \%$, of which 23 were up-regulated and 9 down-regulated. Gene names, count data and Ensembl IDs are shown in Additional file 2: Supplement 2. (XLSX $191 \mathrm{~kb})$

\footnotetext{
Abbreviations

AEC: 3-amino-9-ethyl-carbazole; BMBF: German Ministry of Education and Research; CPS1: Carbamoyl phosphate synthase1; CRC: Colorectal Cancer; CRP: C-reactive protein; CYP: Cytochrome p450; DEG: Differentially expressed genes; ECM: Extracellular matrix; EdgeR: Empirical Analysis of Digital Gene Expression Data in R; EMT: Epithelial-mesenchymal transition; FBS: Foetal bovine serum; FDR: False discovery rate; GO: Gene ontology; HRP: Horseradish peroxidase; IFI205: Interferon-activated gene 205; IFI30: Interferon gamma inducible protein 30; IFITM1: Interferon-induced transmembrane protein 1; IFNa: Interferon-alpha; LogFC: Log fold change; MMP: Matrix metallopeptidase; MMPIs: MMP inhibitors; PBS: Phosphatebuffered saline; PC: Principal component; PCA: Principal component analysis; PCK1: Phosphoenolpyruvate-carboxykinase 1; R: Programming language; RSEM: RNA-seq by Expectation-Maximization; RT: Room temperature;
} 
TEMs: TIE2-expressing monocytes/macrophages; TPM: Transcripts per million; VEGF: Vascular endothelial growth factor

\section{Acknowledgements}

The authors would like to thank Tobias Pukrop and Florian Klemm for their assistance in developing the study design and throughout the process of data retrieval and discussion. They would also like to thank Jetcy Arackal for preparing the CMT-93 cell cultures and Sabine Wolfgramm for her excellent work in preparing the immunolabelling figures for this article. They furthermore express their gratitude to Andrew Entwistle for his critical review of the manuscript.

\section{Ethics approval}

All animal breeding, care, and experimentation procedures were in accordance with the German national and regional legislation on animal protection approved by the Lower Saxony State Office for Consumer Protection and Food Safety (Approval/Reference number: 33.12 42,502-04 - 13/1047).

\section{Funding}

This work was supported by the ebio initiative of the German Ministry of Education and Research (BMBF), DB and AW were funded by the MetastaSys project $(0316173 \mathrm{~A})$ within the ebio initiative. This funding body had no role in the design of the study and collection, analysis, and interpretation of data and in writing the manuscript.

\section{Availability of data and materials}

The datasets used and/or analysed during the current study are available from the corresponding author on reasonable request.

\section{Authors' contributions}

SK directed the study, reviewed data, and wrote the manuscript; DB designed and performed experiments, as well as contributed to the writing of the manuscript; PK coordinated the study and performed experiments, analysed the data and reviewed the manuscript; CH donated the CMT-93 cells and advised on establishing the syngeneic mouse model of liver metastasis; AB contributed to the development of the study design, GS performed the RNA-seq analysis, AW and TB analysed the data and added to the Methods section of the manuscript. All the authors read and approved the final manuscript.

\section{Competing interests}

The authors disclose that there are no conflicts of interest.

\section{Consent for publication}

Not applicable.

\section{Publisher's Note}

Springer Nature remains neutral with regard to jurisdictional claims in published maps and institutional affiliations.

\begin{abstract}
Author details
${ }^{1}$ Department of General, Visceral and Paediatric Surgery, University Medical Centre, Georg - August - University Goettingen, Göttingen, Germany. ${ }^{2}$ Statistical Bioinformatics, Department of Medical Statistics, University Medical Centre, Georg - August - University Goettingen, Göttingen, Germany. ${ }^{3}$ Microarray and Deep-Sequencing Core Facility, Institute for Developmental Biochemistry, University Medical Centre, Georg - August University Goettingen, Göttingen, Germany. ${ }^{4}$ Department of Haematology and Medical Oncology, University Medical Centre, Georg - August University Goettingen, Göttingen, Germany. ${ }^{5}$ Department of Surgery, University Hospital Regensburg, Regensburg, Germany. ${ }^{6}$ Medical Teaching and Medical Education Research, University Hospital Wuerzburg, Julius-Maximilians-University Wuerzburg, Josef-Schneider-Str. 2/D6, 97080 Wuerzburg, Germany.
\end{abstract}

Received: 8 November 2016 Accepted: 11 May 2017 Published online: 19 May 2017

\section{References}

1. Machii R, Saika K. Five-year relative survival rate of colon cancer in the USA, Europe and Japan. Jpn J Clin Oncol. 2014;44(1):105-6.
2. Leporrier J, Maurel J, Chiche L, Bara S, Segol P, Launoy G. A populationbased study of the incidence, management and prognosis of hepatic metastases from colorectal cancer. Br J Surg. 2006;93(4):465-74.

3. Paget $\mathrm{S}$. The distribution of secondary growths in cancer of the breast. Cancer Metastasis Rev. 1989;8(2):98-101.

4. Yokota J. Tumor progression and metastasis. Carcinogenesis. 2000;21(3): 497-503.

5. Bernards R, Weinberg RA. A progression puzzle. Nature. 2002:418(6900):823.

6. Ramaswamy S, Ross KN, Lander ES, Golub TR. A molecular signature of metastasis in primary solid tumors. Nat Genet. 2003;33(1):49-54.

7. Lim B, Mun J, Kim JH, Kim CW, Roh SA, Cho DH, Kim YS, Kim SY, Kim JC. Genome-wide mutation profiles of colorectal tumors and associated liver metastases at the exome and transcriptome levels. Oncotarget. 2015;6(26): 22179-90.

8. Chambers AF, Groom AC, MacDonald IC. Dissemination and growth of cancer cells in metastatic sites. Nat Rev Cancer. 2002;2(8):563-72.

9. Talmadge JE, Fidler IJ. AACR centennial series: the biology of cancer metastasis: historical perspective. Cancer Res. 2010;70(14):5649-69.

10. Clark ME, Smith RR. Liver-directed therapies in metastatic colorectal cancer. J Gastrointest Oncol. 2014;5(5):374-87.

11. Mi K, Kalady MF, Quintini C, Khorana AA. Integrating systemic and surgical approaches to treating metastatic colorectal cancer. Surg Oncol Clin N Am. 2015:24(1):199-214.

12. Dobin A, Davis CA, Schlesinger F, Drenkow J, Zaleski C, Jha S, Batut $P$, Chaisson M, Gingeras TR. STAR: ultrafast universal RNA-seq aligner. Bioinformatics. 2013;29(1):15-21.

13. Li B, Dewey CN. RSEM: accurate transcript quantification from RNA-Seq data with or without a reference genome. BMC Bioinformatics. 2011;12:323.

14. Robinson MD, McCarthy DJ, Smyth GK. edgeR: a Bioconductor package for differential expression analysis of digital gene expression data. Bioinformatics. 2010;26(1):139-40.

15. Alexa A, Rahnenfuhrer J. topGO: topGO: enrichment analysis for Gene ontology. R Packag Version. 2010;2:20,0.

16. Al-Taee KK, Ansari S, Hielscher T, Berger MR, Adwan H. Metastasis-related processes show various degrees of activation in different stages of pancreatic cancer rat liver metastasis. Oncol Res Treat. 2014;37(9):464-70.

17. Fidler IJ. The pathogenesis of cancer metastasis: the 'seed and soil' hypothesis revisited. Nat Rev Cancer. 2003;3(6):453-8.

18. Valastyan S, Weinberg RA. Tumor metastasis: molecular insights and evolving paradigms. Cell. 2011;147(2):275-92.

19. Gupta GP, Massague J. Cancer Metastasis: building a framework. Cell. 2006; 127(4):679-95.

20. Bissell MJ, Hines WC. Why don't we get more cancer? A proposed role of the microenvironment in restraining cancer progression. Nat Med. 2011; 17(3):320-9.

21. Wen SW, Ager El, Christophi C. Bimodal role of Kupffer cells during colorectal cancer liver metastasis. Cancer Biol Ther. 2013;14(7):606-13.

22. Joyce JA, Pollard JW. Microenvironmental regulation of metastasis. Nat Rev Cancer. 2009:9(4):239-52.

23. Lovering RC, Camon EB, Blake JA, Diehl AD. Access to immunology through the Gene ontology. Immunology. 2008;125(2):154-60.

24. Becht E, de Reynies A, Giraldo NA, Pilati C, Buttard B, Lacroix L, Selves J, Sautes-Fridman C, Laurent-Puig P, Fridman WH. Immune and stromal classification of colorectal cancer is associated with molecular subtypes and relevant for precision immunotherapy. Clin Cancer Res. 2016;22(16): 4057-66.

25. Hamilton TD, Leugner D, Kopciuk K, Dixon E, Sutherland FR, Bathe OF. Identification of prognostic inflammatory factors in colorectal liver metastases. BMC Cancer. 2014;14:542.

26. Catarinella M, Monestiroli A, Escobar G, Fiocchi A, Tran NL, Aiolfi R, Marra P, Esposito A, Cipriani F, Aldrighetti L, et al. IFNalpha gene/cell therapy curbs colorectal cancer colonization of the liver by acting on the hepatic microenvironment. EMBO Mol Med. 2016;8(2):155-70.

27. Nadal C, Maurel J, Gascon P. Is there a genetic signature for liver metastasis in colorectal cancer? World J Gastroenterol. 2007;13(44):5832-44.

28. van der Wal GE, Gouw AS, Kamps JA, Moorlag HE, Bulthuis ML, Molema $\mathrm{G}$, de Jong KP. Angiogenesis in synchronous and metachronous colorectal liver metastases: the liver as a permissive soil. Ann Surg. 2012; 255(1):86-94

29. Kuramochi H, Hayashi K, Uchida K, Miyakura S, Shimizu D, Vallbohmer D, Park S, Danenberg KD, Takasaki K, Danenberg PV. Vascular endothelial 
growth factor messenger RNA expression level is preserved in liver metastases compared with corresponding primary colorectal cancer. Clin Cancer Res. 2006;12(1):29-33.

30. Kocakova I, Melichar B, Kocak I, Bortlicek Z, Buchler T, Dusek L, Petruzelka L, Kohoutek M, Prausova J, Finek J, et al. Bevacizumab with FOLFIRI or XELIRI in the first-line therapy of metastatic colorectal carcinoma: results from Czech observational registry. Anticancer Res. 2015;35(6):3455-61.

31. Yamazaki K, Nagase M, Tamagawa H, Ueda S, Tamura T, Murata K, Eguchi Nakajima T, Baba E, Tsuda M, Moriwaki T, et al. Randomized phase III study of bevacizumab plus FOLFIRI and bevacizumab plus MFOLFOX6 as first-line treatment for patients with metastatic colorectal cancer (WJOG4407G). Ann Oncol. 2016;27(8):1539-46.

32. Zeng ZS, Shu WP, Cohen AM, Guillem JG. Matrix metalloproteinase-7 expression in colorectal cancer liver metastases: evidence for involvement of MMP-7 activation in human cancer metastases. Clin Cancer Res. 2002:8(1):144-8.

33. Lee SK, Han YM, Yun J, Lee CW, Shin DS, Ha YR, Kim J, Koh JS, Hong SH, Han DC, et al. Phosphatase of regenerating liver-3 promotes migration and invasion by upregulating matrix metalloproteinases-7 in human colorectal cancer cells. Int J Cancer. 2012;131(3):E190-203.

34. Ochiai H, Nakanishi Y, Fukasawa Y, Sato Y, Yoshimura K, Moriya Y, Kanai Y, Watanabe $\mathrm{M}$, Hasegawa $\mathrm{H}$, Kitagawa $\mathrm{Y}$, et al. A new formula for predicting liver metastasis in patients with colorectal cancer: immunohistochemical analysis of a large series of 439 surgically resected cases. Oncology. 2008; 75(1-2):32-41

35. Fang YJ, Lu ZH, Wang GQ, Pan ZZ, Zhou ZW, Yun JP, Zhang MF, Wan DS. Elevated expressions of MMP7, TROP2, and survivin are associated with survival, disease recurrence, and liver metastasis of colon cancer. Int I Color Dis. 2009;24(8):875-84.

36. Wagenaar-Miller RA, Gorden L, Matrisian LM. Matrix metalloproteinases in colorectal cancer: is it worth talking about? Cancer Metastasis Rev. 2004; 23(1-2):119-35.

37. Shen F, Cai WS, Li JL, Feng Z, Liu QC, Xiao HQ, Cao J, Xu B. Synergism from the combination of ulinastatin and curcumin offers greater inhibition against colorectal cancer liver metastases via modulating matrix metalloproteinase-9 and E-cadherin expression. Onco Targets Ther. 2014; 7:305-14.

\section{Submit your next manuscript to BioMed Central and we will help you at every step:}

- We accept pre-submission inquiries

- Our selector tool helps you to find the most relevant journal

- We provide round the clock customer support

- Convenient online submission

- Thorough peer review

- Inclusion in PubMed and all major indexing services

- Maximum visibility for your research

Submit your manuscript at www.biomedcentral.com/submit

) Biomed Central 